\title{
A IMPORTÂNCIA DOS SOFT SKILLS NAS DESCRIÇÕES DAS VAGAS DE GERENTE DE PROJETOS DE TI
}

THE IMPORTANCE OF SOFT SKILLS IN JOB DESCRIPTIONS FOR IT PROJECT MANAGER

\author{
LA IMPORTANCIA DE LOS SOFT SKILLS EN LAS DESCRIPCIONES DE LAS VACANTES DE GERENTE DE \\ PROYECTOS DE TI
}

\author{
NADIA PEDROSA \\ Doutoranda \\ Universidade Nove de Julho - Brasil \\ nadiapedrosa@uninove.edu.br \\ ORCID: http://orcid.org/0000-0003-3865-3093 \\ LUCIANO FERREIRA DA SILVA \\ Doutor \\ Universidade Nove de Julho - Brasil \\ prof.lfs7725@gmail.com \\ ORCID: https://orcid.org/0000-0001-6482-8729
}

Submetido em: 12/10/2018

Aprovado em: 18/02/2019

Doi: alcance.v26n1(Jan/Abr).p045-060

\section{RESUMO}

A importância estratégica de projetos requer a contratação de gerentes de projetos de $\mathrm{Tl}$ (Tecnologia da Informação) que atendam à agenda de requisitos requeridos pelas organizações. A literatura preconiza que há uma relação positiva entre as competências do gerente de projetos e os resultados do projeto. Os soft skills ou habilidades interpessoais compõem parte de tais competências. Nesse sentido, cada vez mais os soft skills do gerente de projetos são percebidos como habilidades essenciais para as organizações que visam à multiplicação de seus resultados. $O$ objetivo deste artigo é identificar quais são os principais soft skills solicitados nos anúncios de vagas on-line de gerente de projetos de TI no Brasil. Optou-se pela abordagem qualitativa, cujo método de pesquisa adotado envolveu uma análise de frequência e de conteúdo das descrições das vagas para confrontar os dados provenientes da literatura e os resultados desta pesquisa. A contribuição pretendida com este trabalho é oferecer uma lista dos soft skills mais citados nas vagas de gerentes de projetos de TI no Brasil.

Palavras-chave: Gerente de projetos. TI. Soft skills. Contratação. Vagas.

\begin{abstract}
Due to the strategic importance of projects, Information Technology (IT) project managers must be hired that meet the organization's list of requirements. A review of the literature suggests that there is a positive relationship between the competencies of the project manager and the outcomes of the project. Soft skills, or interpersonal skills, are part of these competencies. These skills are increasingly perceived as essential for IT project managers in organizations seeking to improve their results. This article aims to identify which soft skills are requested in vacancy advertisements for the position of IT project manager. We opted for the qualitative approach; the research method involved analysis of frequency and content of job descriptions, comparing the research results with the data found in the literature. It is hoped that this paper will enable a list to be produced, of the most frequently cited soft skills in the Brazilian vacancy advertisements for IT project managers.
\end{abstract}


Keywords: Project Manager. IT. Soft skills. Hiring. Vacancies.

\section{RESUMEN}

La importancia estratégica de los proyectos requiere la contratación de gerentes de proyectos de TI (Tecnologías de la Información) que atiendan la agenda de requisitos requeridos por las organizaciones. La literatura preconiza que hay una relación positiva entre las competencias del gerente de proyectos y los resultados del proyecto. Los soft skills o habilidades interpersonales forman parte de tales competencias. En este sentido, cada vez más los soft skills del gerente de proyectos se percibe como habilidades esenciales para las organizaciones que apuntan a la multiplicación de sus resultados. El objetivo de este artículo es identificar cuáles son los principales soft skills solicitadas en los anuncios de vacantes online de gerente de proyectos de TI. Se optó por el abordaje cualitativo cuyo método de investigación adoptado implicó un análisis de frecuencia y de contenido de las descripciones de las vacantes para confrontar los datos provenientes de la literatura y los resultados de esta investigación. La contribución pretendida con este trabajo es ofrecer una lista de los soft skills más citados en las vacantes de gerentes de proyectos de TI en Brasil.

Palabras clave: Gerente de proyectos. TI. Soft skills. Contratación. Vacantes.

\section{INTRODUÇÃO}

O fenômeno da projetização, que é quando a estrutura organizacional de uma empresa baseia-se em projetos, é o resultado do aumento dos padrões de qualidade do produto e da crescente pressão pelo cumprimento de prazos e custos dentro das organizações, que enfrentam esses desafios por meio da abertura de projetos (Geithner \& Menzel, 2016). De acordo com Gray e Ulbrich (2017), a criação de valor ao negócio de uma organização depende estreitamente de projetos bem-sucedidos que atinjam os benefícios esperados. Pesquisas apontam que, no oeste europeu, um terço das atividades econômicas é conduzido dentro do escopo de projetos (Schoper, Wald, Ingason \& Fridgeirsson, 2018). Portanto, os investimentos em projetos têm sido uma mola propulsora para as organizações que buscam o sucesso.

No Brasil, o uso da TI (Tecnologia da Informação) viabiliza e disponibiliza produtos e serviços inovadores para os clientes de diferentes segmentos de negócio. Se por um lado os consumidores finais ganham em qualidade e benefícios na experiência com produtos e serviços inovadores; por outro, as organizações elevam a sua produtividade e especialização, o que as fazem investir mais e mais em projetos de TI para garantir a competitividade (Elliott \& Dawson, 2015). Segundo a Deloitte (2017), mesmo em tempos de crise, o setor bancário brasileiro investiu $\mathrm{R} \$ 18,6$ bilhões em tecnologia no período de 2016.

As organizações estimam que seus projetos deem retorno, e é neste contexto que o gerente de projetos vem conquistando um patamar de importância para conferir bom desempenho aos projetos. Com o avanço na disciplina de gerenciamento de projetos, a figura do gerente de projetos ganha cada vez mais relevância. $\mathrm{Na}$ literatura, não raro, se observam autores que citam a relação positiva das competências do gerente de projetos de TI sobre os fatores críticos de sucesso do projeto (Stevenson \& Starkweather, 2010).

A combinação de hard skills com o soft skills em termos de habilidades se tornou uma vantagem competitiva para o gerente de projetos. Pode-se dizer que hard skills no contexto de gerenciamento de projetos são as competências técnicas, tais como: habilidade em planejar o projeto; análise de risco; monitoramento e controle do prazo do projeto e perícia no uso de ferramentas de gestão de projetos (Geithner \& Menzel, 2016). Já os soft skills, ou também denominados habilidades humanas ou competências comportamentais, podem ser exemplificados como: liderança orientada a metas; habilidade em tomar decisões; gestão de conflitos; construção de equipes e facilidade em trabalhar em equipe (Geithner \& Menzel, 2016).

Ao contratarem os seus profissionais de $\mathrm{TI}$, as empresas antes mantinham seu foco somente nos hard skills (Joseph, Ang, Chang \& Slaughter, 2010). Porém essa realidade mudou e já se sabe que os hard skills não são mais suficientes para o sucesso dos projetos da TI (Ahmed, Capretz \& Campbell, 2012). Agora, os soft skills se tornaram condição sine qua non para alavancar os resultados da TI (Joseph et al., 2010). Neste contexto, este artigo procurará explorar o universo dos soft skills do gerente de projetos de TI. Dada a importância do gerente de projetos para o sucesso de projetos de TI, torna-se evidente que não contratar gerentes de projetos com soft skills 
pode ser prejudicial para a saúde financeira das organizações (Ahsan, Ho \& Khan, 2013). Principalmente quando se sabe que $91 \%$ dos projetos de TI falham em grandes empresas (The Standish Group, 2014).

Em projetos de $\mathrm{Tl}$, qualquer que seja a entrega, haverá sempre o envolvimento do aspecto humano (Ahmed et al., 2012). Por esse motivo, esses mesmos autores defendem a ideia de que projetos de $\mathrm{Tl}$ atingem melhores resultados quando a equipe de projetos possui soft skills (Ahmed et al., 2012). Conforme descrito anteriormente, soft skills referem-se às habilidades humanas. Devido ao viés estritamente comportamental relacionado ao conceito soft skill, surge uma condição preocupante quanto à importância que os softs skills têm nas contratações, principalmente no que se refere às descrições das vagas de gerentes de projetos de TI. Com efeito, a questão de pesquisa que se quer responder neste estudo é: Quais são os soft skills mais citados nas descrições de vagas de gerente de projetos de TI no Brasil?

Pela própria problemática da pesquisa, estima-se atingir um público composto por profissionais de $\mathrm{TI}$. Gerentes de projetos de TI, principalmente, interessados em entender quais são os soft skills que estão em roga nas descrições das vagas. Tal problemática leva ao objetivo principal deste artigo, que é identificar quais são os principais soft skills solicitados nos anúncios de vagas on-line de gerente de projetos de TI.

Não obstante o tema seja alvo de pesquisas até os dias atuais, os registros de estudos específicos sobre quais os soft skills do gerente de projetos de TI aparecem nas descrições das vagas são modestos. $O$ trabalho de Walter Saunders, Nunes e De Carvalho (2018) realizou uma bibliometria com 88 artigos sobre as competências do gerente de projetos e, após a análise de conteúdo, os autores concluíram que somente $8 \%$ da literatura pesquisada usaram anúncios de vagas de gerentes de projetos como procedimentos de coleta de dados. Pilati e Araújo (2008) reconhecem que os trabalhos acadêmicos sobre o processo de contratação no Brasil são escassos, embora a prática de contratar seja frequente. Logo, a carência de trabalhos específicos na literatura culminou com a escolha do presente tema de pesquisa.

$O$ alvo desta pesquisa recai sobre o gerente de projetos de TI. Pois, segundo Walter Saunders et al. (2018), o setor de TI é o que gera a maior demanda de vagas de gerente de projetos no Brasil. Em países como Estados Unidos e Canadá, essa realidade se repete conforme o relatório publicado pela Bullhornreach (2014), que diz que a maioria das vagas postadas em mídias sociais destes dois países pertence à indústria de TI.

Este trabalho possui as seguintes seções: (1) Introdução aqui finalizada; (2) Referencial teórico, que contempla os conceitos dos principais pilares teóricos do trabalho; (3) Procedimentos Métodológicos, com o detalhe das técnicas adotadas para a pesquisa; (4) Resultados e Discussões dos resultados extraídos da pesquisa de campo frente à literatura; e (5) Conclusão do artigo.

\section{REFERENCIAL TEÓRICO}

As competências de pessoas podem ser divididas em duas categorias de habilidades: as técnicas (hard skills) e as pessoais (soft skills) (Bolli \& Renold, 2015; Wikle \& Fagin, 2015). Skill é uma palavra inglesa que significa: (1) "o conhecimento e a habilidade que permite você fazer algo bem" e (2) "é um tipo de trabalho ou ofício que requer um treinamento especial e conhecimento" (Collins, 1992, p. 748). Portanto, os hard skills são competências especializadas para que o indivíduo realize uma tarefa, os quais podem ser adquiridos por meio de treinamento, educação ou experiência (Wikle \& Fagin, 2015). Já os soft skills são competências genéricas, tais como comunicação efetiva e habilidade em atuar em ambiente colaborativo (Wikle \& Fagin, 2015).

Sob uma visão holística, tem-se que o hard skill é a habilidade técnica específica que se relaciona com um determinado campo de conhecimento, já o soft skill é composto por atributos de personalidade e de comportamento (Berbegal-Mirabent, Gil-Doménech \& Alegre, 2017). Mateo et al. (2017) afirmam que o gerente de projetos em seu ambiente de trabalho precisa ter não somente os hard skills, mas também os soft skills para lidar com objetivos conflitantes. Autores como Bolli e Renold (2015) e Creasy e Anantatmula (2013) são partidários de que os soft skills são mais importantes que os hard skills. Os resultados alcançados por Livesey (2016), em sua pesquisa usando o método Delphi com gerentes de projetos com orçamentos para projetos acima dos US $\$ 500$ milhões de dólares, concluiu que o aumento da necessidade por soft skills é mais pronunciada do que o aumento da necessidade por hard skills. Por este motivo, o presente artigo focalizará no estudo dos soft skills do gerente de projetos de TI. 


\subsection{DEFINIÇÃO DE SOFT SKILLS}

Definir precisamente o conceito de soft skills não é uma tarefa fácil devido às várias nomenclaturas associadas a esse termo. Behaviour skills ou habilidades comportamentais, generic skills ou habilidades genéricas, core skills ou habilidades essenciais, personal skills ou habilidades pessoais e key skills ou habilidades-chave são algumas terminologias citadas por Dench (1997). Esse autor usa frequentemente o termo habilidades pessoais ou personal skills como sendo habilidades inatas ligadas ao comportamento do indivíduo. Já Elliott e Dawson (2015) empregam o termo interpersonal skills ou habilidades interpessoais. Soft competences ou competências soft é a expressão usada por Skulmoski e Hartman (2009). El-Sabaa (2001) recomenda adotar o termo human skills ou habilidades humanas, que são demonstradas sob a forma de como o gerente de projetos responde às atitudes de seus superiores, pares, subordinados e como ele se comporta diante destas partes interessadas. E, por último, administrative skills ou habilidades administrativas é como os autores Pinto, Patanakul e Pinto (2017) denominam soft skills.

Tabela 1.

Resumo da nomenclatura usada para referenciar soft skills

\begin{tabular}{l|l}
\hline Autores & Nomenclatura de soft skills \\
\hline Dench (1997) & - behaviour skills ou habilidades comportamentais \\
& - generic skills ou habilidades genéricas \\
& - core skills ou habilidades essenciais \\
& - personal skills ou habilidades pessoais \\
& - key skills ou habilidades-chave \\
\hline Elliott e Dawson (2015) & - interpersonal skills ou habilidades interpessoais \\
\hline Skulmoski e Hartman (2009) & - soft competences ou competências soft \\
\hline El-Sabaa (2001) & - human skills ou habilidades humanas \\
\hline Joseph et al. (2010) & - soft skills \\
\hline Pinto, Patanakul e Pinto (2017) & - administrative skills ou habilidades administrativas \\
\hline
\end{tabular}

Fonte: Elaborada pelos autores com base na literatura citada na coluna Autores.

A Tabela 1 reflete o resumo do glossário usado para referenciar soft skills na literatura consultada. Por convenção, este trabalho optou pelo uso do termo soft skill por ser este um termo genérico (Joseph et al., 2010) e que é bem compreendido tanto no meio acadêmico quanto no corporativo.

Os soft skills podem ser compreendidos como habilidades interpessoais (Bolli \& Renold, 2015; Gray \& Ulbrich, 2017; Skulmoski \& Hartman, 2009). Já para Livesey (2017), soft skills são as habilidades que envolvem a gestão de pessoas. Em seu artigo, Turner (2016) descreve que os soft skills são um insight sobre as qualidades necessárias para o gerente de projetos ser bem-sucedido. Para El-Sabaa (2001), soft skills são as habilidades em lidar com os aspectos humanos e, segundo suas pesquisas, possuem influência sobre o sucesso dos projetos, bem mais que os hard skills. Há quem denomine soft skill como competências transversais que são as competências não técnicas, como: comunicação e autonomia (Soares, Sepúlveda, Monteiro, Lima \& DinisCarvalho, 2013).

Existem autores que corroboram com a ideia de que os soft skills estariam relacionados à personalidade do indivíduo. Nesse aspecto, é possível citar Ahmed et al. (2012), que definem soft skills como habilidades ligadas aos traços da personalidade e às atitudes do indivíduo e que dirigem o seu comportamento. Comunicação, cooperação, liderança, independência e criatividade são exemplos de soft skills para Balcar et al. (2018).

A maioria dos autores, entretanto, associa o termo soft skill às pessoas no âmbito corporativo. Sukhoo, Barnard, Eloff e Van der Poll (2005) defendem a ideia de que soft skill é uma arte que está relacionada à gestão e ao trabalho com pessoas, com o objetivo final de alcançar a satisfação do cliente e criar um ambiente favorável para que o time do projeto entregue produtos de qualidade dentro do prazo e dos custos acordados. Belzer (2001) também descreve soft skill como uma arte e o define como habilidades não técnicas. Para Andoh-Baidoo et al. (2011), soft skill é definido como uma habilidade fundamental que não está vinculada a uma determinada tarefa, mas sim a uma capacidade latente que um indivíduo tem para alcançar resultados em certos tipos de tarefas. Lent e Pinkowska (2012) reconhecem que soft skill é uma agregação de todas as habilidades cognitivas e pessoais, 
que contribuem para uma maior eficiência da execução dos processos relacionados ao fator humano do gerenciamento de projetos.

Tabela 2.

Resumo das definições de soft skills

\begin{tabular}{|c|c|}
\hline Autores & Definições de soft skills \\
\hline Livesey (2017) & - Habilidades que envolvem a gestão de pessoas. \\
\hline Turner (2016) & $\begin{array}{l}\text { - Insight sobre as qualidades necessárias para o gerente de projetos ser bem- } \\
\text { sucedido. }\end{array}$ \\
\hline El-Sabaa (2001) & $\begin{array}{l}\text { - Habilidades em lidar com os aspectos humanos e, segundo suas pesquisas, } \\
\text { possuem influência sobre o sucesso dos projetos, bem mais que os hard skills. }\end{array}$ \\
\hline $\begin{array}{l}\text { Soares, Sepúlveda, Monteiro, Lima e } \\
\text { Dinis-Carvalho (2013) }\end{array}$ & $\begin{array}{l}\text { - Competências transversais que são as competências não técnicas, como: } \\
\text { comunicação e autonomia; } \\
\text { - Usam o gerenciamento de projetos como um exemplo de soft skills dentro da } \\
\text { engenharia. }\end{array}$ \\
\hline Ahmed et al. (2012) & $\begin{array}{l}\text { - Habilidades ligadas aos traços da personalidade e às atitudes do indivíduo e que } \\
\text { dirigem o seu comportamento. }\end{array}$ \\
\hline $\begin{array}{l}\text { Sukhoo, Barnard, Eloff e Van der Poll } \\
(2005)\end{array}$ & $\begin{array}{l}\text { - Uma arte que está relacionada à gestão e ao trabalho com pessoas, com o objetivo } \\
\text { final de alcançar a satisfação do cliente e criar um ambiente favorável para que o } \\
\text { time do projeto entregue produtos de qualidade dentro do prazo e do custo } \\
\text { acordados. }\end{array}$ \\
\hline Belzer (2001) & - Uma arte e o define como habilidades não técnicas. \\
\hline Andoh-Baidoo et al. (2011) & $\begin{array}{l}\text { - Habilidade fundamental que não está vinculada a uma determinada tarefa, mas } \\
\text { sim a uma capacidade latente que um indivíduo tem para alcançar resultados em } \\
\text { certos tipos de tarefas. }\end{array}$ \\
\hline Lent e Pinkowska (2012) & $\begin{array}{l}\text { - Uma agregação de todas as habilidades cognitivas e pessoais, que contribuem } \\
\text { para uma maior eficiência da execução dos processos relacionados ao fator humano } \\
\text { do gerenciamento de projetos. }\end{array}$ \\
\hline
\end{tabular}

Fonte: Elaborada pelos autores com base na literatura citada na coluna Autores.

A Tabela 2 consolida as definições de soft skill a partir da literatura pesquisada. Para este trabalho, estipulou-se a definição de habilidades interpessoais dos autores Bolli e Renold (2015); Gray e Ulbrich (2017); Skulmoski e Hartman (2009) como convenção.

\subsection{SOFT SKILLS DO GERENTE DE PROJETOS DE TI}

A importância dos soft skills no gerenciamento de projetos depende da natureza do projeto. Por exemplo, projetos com times geograficamente dispersos introduzem problemas adicionais e, portanto, soft skills como: clareza na comunicação e na habilidade de desenvolver confiança são mais demandados (Livesey, 2016). Já as empresas de inovação exigem em maior grau as habilidades em relacionar-se com clientes (Danneels, 2000). Para este trabalho, o enfoque será dado para os soft skills do gerente de projetos da área de TI.

$\mathrm{O}$ crescimento acelerado da indústria de Tl é um desafio para o gerente de projetos de TI (Zahra, Nazir \& Khalid, 2014). Novos dispositivos eletrônicos, novas plataformas e novos modelos são lançados todos os meses como resultados de projetos bem-sucedidos em TI (Zahra et al., 2014). Para esta pesquisa, o conceito de projetos de TI está relacionado a: desenvolvimento e melhorias de software, integração entre sistemas, aquisições em TI, manutenção, infraestrutura, rede e projetos relacionados à segurança da informação (Keil, Lee \& Deng, 2013). Devido à relação positiva entre as habilidades do gerente de projetos de Tl e o sucesso dos projetos de $\mathrm{Tl}$ (Keil et al., 2013), muitos pesquisadores têm se dedicado ao estudo sobre os soft skills do gerente de projetos.

Seguindo nesta direção, muitos autores associam quais são os soft skills importantes para o bom desempenho do gerente de projetos de TI. Kliem (1991), por exemplo, elenca os soft skills do gerente de projetos de TI em seis categorias: (1) habilidades de comunicação; (2) habilidades de gestão de equipes; (3) habilidades interpessoais; (4) habilidades interpretativas; (5) habilidades de gestão de crise; e (6) habilidades de gestalt. Entretanto, cada categoria pode demandar uma série de outros soft skills. Já Sukhoo et al. (2005) contribuem com

Revista Alcance - Eletrônica - vol. 26 - n. 1 - Jan./Abr. 2019 
um estudo que lista os soft skills do gerente de projetos de TI, como: comunicação; construção de equipes; flexibilidade; criatividade; liderança; gerenciamentos de estresse e de conflito são mais relevantes em cada fase do ciclo de vida da gestão de projetos: iniciação, planejamento, execução, controle e encerramento.

Ainda sobre os soft skills dos gerentes de projetos de TI, Pant e Baroudi (2008) citam habilidades em comunicação externa, satisfação do cliente, gerenciamento de conflitos e gerenciamento e motivação da equipe do projeto. Esses mesmos autores destacam a importância da habilidade de relacionar-se como complemento dos hard skills, porque os resultados do projeto são conquistados por meio das pessoas, com seus relacionamentos e habilidades, e não pelo simples uso de técnicas ou conhecimentos (Pant \& Baroudi, 2008). Belzer (2001) advoga a favor de que os soft skills dos gerentes de projetos sejam peças fundamentais da entrega bem-sucedida do projeto e cita comunicação, efetividade organizacional, liderança, solução de problemas, tomada de decisão, construção de equipe, flexibilidade, criatividade e confiabilidade como importantes soft skills no gerenciamento de projetos. Em cada uma das listas citadas, nota-se que há uma convergência na opinião dos autores ao definirem os soft skills dos gerentes de projetos de TI. Por exemplo: comunicação, gerenciamento de conflito e liderança foram citados repetidamente por diferentes pesquisadores.

Stevenson e Starkweather (2010) investigaram e ordenaram as competências humanas do gerente de projetos de TI mais importantes para a obtenção do sucesso em projetos nas indústrias americanas. O resultado da pesquisa constatou que de 15 competências identificadas, as seis mais importantes são: (1) liderança; (2) habilidade de comunicação em múltiplos níveis organizacionais; (3) habilidade verbal; (4) habilidade escrita; (5) atitude; e (6) capacidade em lidar com ambiguidade e mudança. Esse resultado demonstra que os executivos de TI norte-americanos preferem os soft skills em contraste com os hard skills. A Tabela 3 apresenta um resumo das definições teóricas destes soft skills referenciados no trabalho de Stevenson e Starkweather (2010).

Tabela 3.

Descrição dos principais soft skills do gerente de projetos de TI

\begin{tabular}{l|l}
\hline Soft skills & Definição \\
\hline Liderança & $\begin{array}{l}\text { Habilidade em que os líderes engajam, influenciam, inspiram e guiam os } \\
\text { outros para cumprir objetivos; efetivamente representam a equipe ou a } \\
\text { organização para um grupo de partes interessadas (Schoreder, 2014). }\end{array}$ \\
\hline $\begin{array}{l}\text { Habilidade de comunicação em múltiplos níveis } \\
\text { organizacionais }\end{array}$ & $\begin{array}{l}\text { Habilidade em comunicar problemas complexos para todos os níveis da } \\
\text { cadeia de comando (Kliem, 1991). }\end{array}$ \\
\hline Habilidade verbal & $\begin{array}{l}\text { Habilidade em escolher palavras concretas, ou seja, palavras que } \\
\text { transmitem significado específico e que sejam do conhecimento do } \\
\text { receptor (Gillard, 2017). }\end{array}$ \\
\hline Atitude & $\begin{array}{l}\text { Habilidade em reiterar os objetivos da equipe; escrever resumos e planos } \\
\text { de ação como resultados de teleconferências (Gillard, 2017). }\end{array}$ \\
\hline $\begin{array}{l}\text { Capacidade em lidar com ambiguidade e e encrita } \\
\text { mudança }\end{array}$ & $\begin{array}{l}\text { Habilidade em posicionar-se diante de uma tomada de decisão ou } \\
\text { aceitação a um risco (Schoreder, 2014). }\end{array}$ \\
\hline $\begin{array}{l}\text { Habilidade em estar aberto para a mudança e para o novo; adaptar 0 } \\
\text { comportamento ou trabalhar métodos para mudar circunstâncias, } \\
\text { obstáculos ou nova informação (Schoreder, 2014). }\end{array}$ \\
\hline
\end{tabular}

Fonte: Elaborada pelos autores com base em Schoreder (2014); Gillard (2017); Stevenson e Starkweather (2010) e Kliem (1991).

A pesquisa de campo deste estudo fomenta a investigação de quais softs skills do gerente de projetos de TI mais se apresentaram nas descrições das vagas de emprego. A pesquisa usou como base comparativa 0 artigo intitulado "PM critical competency index: IT execs prefer soft skills" de Stevenson e Starkweather (2010). A escolha deste trabalho específico se justifica por este ser o mais citado na base indexadora Web of Science, com 44 citações; enquanto que o segundo artigo mais citado é o de Creasy e Anantatmula (2013), com 26 citações, conforme consulta realizada pelos pesquisadores em 26 de maio de 2018 com a cadeia de busca, campo tópico = "soft skill" e tópico = "project manage". 


\section{PROCEDIMENTOS METODOLÓGICOS}

Nesta seção, introduzem-se o método de pesquisa adotado para este trabalho, a justificativa para tal escolha e o racional usado pelos autores que explica os procedimentos metodológicos desta pesquisa.

\subsection{DELINEAMENTO DA PESQUISA}

O objetivo central da pesquisa é identificar quais são os principais soft skills mais solicitados nos anúncios de vagas on-line de gerente de projetos de TI no Brasil. Portanto, a unidade de análise é composta pelas descrições das vagas. Dada a natureza investigativa da pesquisa, optou-se pela abordagem qualitativa, ideal para a realização de estudos exploratórios como este, segundo Creswell (2010).

\subsection{PROCEDIMENTOS DE COLETA DE DADOS}

O primeiro estágio da coleta de dados foi realizado no site de vagas Linkedln. A escolha dessa plataforma foi motivada pelo fato de o Linkedln ser a maior rede social profissional do mundo e contar com mais de 450 milhões de usuários em 24 idiomas (Tifferet \& Vilnai-Yavetz, 2018). Segundo a revista Exame (2016), o Brasil é 0 terceiro país do mundo mais presente no Linkedln, com 25 milhões de usuários ativos, ficando atrás somente dos Estados Unidos e da Índia. O Linkedln é especializado em redes profissionais, soluções de recrutamento e busca de emprego (Girard \& Fallery, 2010). Para atender a essa última funcionalidade - busca de emprego -, o Linkedln oferece um serviço pago pelo recrutador que deseja publicar anúncios de vagas no site. $O$ usuário do Linkedln tem acesso a mais de 10 milhões de vagas anunciadas em 200 países e tem à disposição dados de mais de 9 milhões de empresas (Veja, 2017).

Ao acessar o endereço eletrônico https://www.linkedin.com/jobs/, há dois campos livres para digitação: (1) pesquisar vagas e (2) pesquisar localidade, conforme pode ser visto na Figura 1. Estes campos servem para filtrar as consultas de vagas de emprego no Linkedln pelo título da vaga e pela sua localização, respectivamente. Para ampliar a amostra de dados, os pesquisadores fizeram quatro consultas com quatro títulos de vagas diferentes: "gerentes de projetos", "líder de projetos", "coordenador de projetos" e "project manager" e manteve a localidade igual a "Brasil". A opção de uso pela palavra-chave em inglês "project manager" se deu porque esse termo é também usado como título de vaga de gerentes de projetos no Brasil, mesmo quando a descrição da vaga está no idioma português.

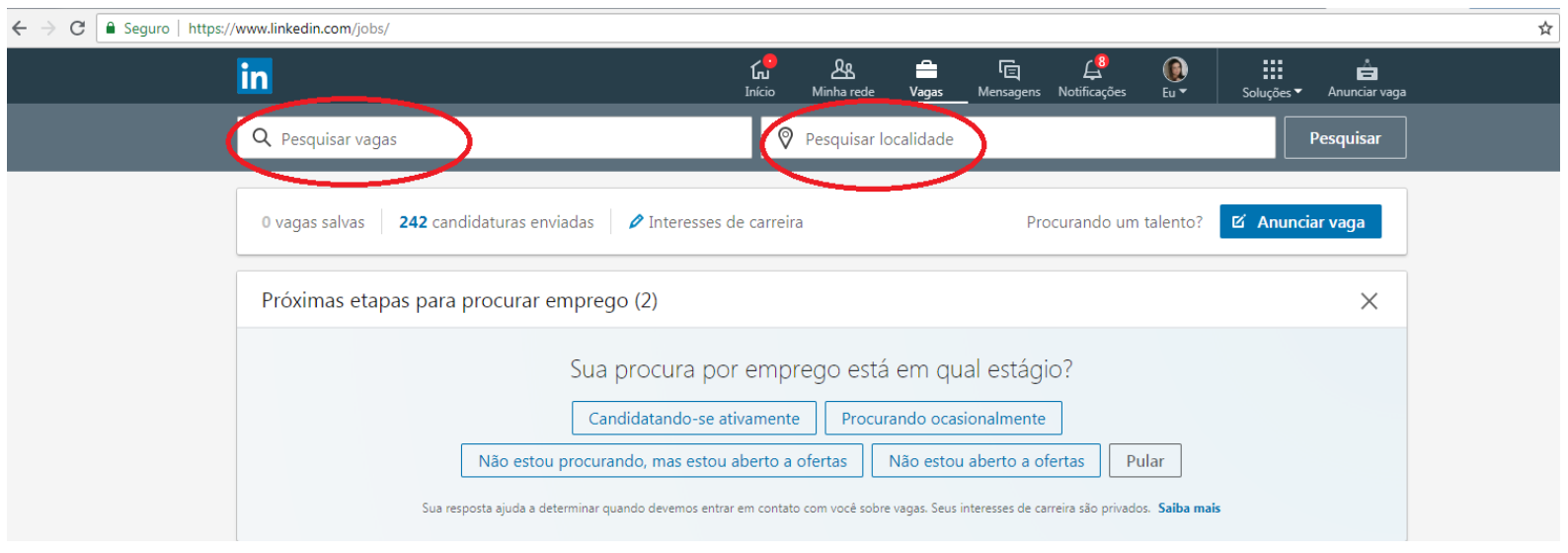

Figura 1. Página de pesquisa de vagas no Linkedln

Fonte: Elaborada pelos autores com base no site Linkedln

As consultas no site Linkedln foram realizadas entre os dias 20 de julho de 2018 e 21 de julho de 2018. A busca com a palavra-chave "gerente de projetos" retornou 879 vagas; já o título "líder de projetos" gerou 64 resultados; com "coordenador de projetos", apareceram 238 vagas; e "project manager" exibiu 168 vagas; totalizando 1.349 vagas. Esses são os números finais das consultas demonstrados no Linkedln com base no segundo dia de pesquisa. 
Entre o primeiro e o segundo dias da consulta, o site apresentou uma oscilação no número de vagas resultante da pesquisa, haja vista a inclusão e a exclusão de vagas no site pelos recrutadores ser algo dinâmico e on-line, o que pode acontecer a qualquer momento. Isso justifica a diferença entre a quantidade de vagas iniciais e a quantidade de vagas finais exibidas no resultado das pesquisas.

O processo de coleta foi manual e se caracterizou pela cópia do texto da descrição da vaga resultante da consulta no site do Linkedln em uma planilha eletrônica. Tal procedimento foi repetido vaga a vaga. Entretanto, durante a coleta de dados, e devido à oscilação das vagas no site, o total de vagas sofreu uma diferença. Neste ínterim, a quantidade total de vagas mudou de 1.349 para as efetivas 1.368 descrições de vagas, que constituíram os dados secundários desta pesquisa. É o que mostra a Tabela 4.

Tabela 4.

Informações das consultas de vagas realizadas no Linkedln

\begin{tabular}{l|l|l|l}
\hline Título da vaga & Data da Consulta & $\begin{array}{l}\text { Quantidade de vagas } \\
\text { iniciais }\end{array}$ & Quantidade de vagas finais \\
\hline "gerente de projetos" & $20 / 07 / 2018$ & 871 & 879 \\
\hline "líder de projetos" & $21 / 07 / 2018$ & 64 & 64 \\
\hline "coordenador de projetos" & $21 / 07 / 2018$ & 234 & 238 \\
\hline "project manager" & $21 / 07 / 2018$ & 169 & 168 \\
\hline \multicolumn{2}{l}{ Total de vagas apresentadas no site } & 1338 & 1349 \\
\hline \multicolumn{2}{l}{ Total da captação de vagas } & & 1368 \\
\hline
\end{tabular}

Fonte: Elaborada pelos autores.

\subsection{PROCEDIMENTOS DE ANÁLISE DE DADOS}

A pesquisa qualitativa geralmente coloca os números em segundo plano, porque sua ótica está mais na análise das qualidades essenciais dos dados do que na análise numérica (Miles, Huberman \& Saldaña, 1994). Porém, a contagem dos incidentes pode ser um elemento relevante, a partir de dados acumulados, do que é importante, significativo ou recorrente (Miles et al., 1994). Nesse sentido, o presente trabalho fez uso de contagens para identificar quais são os soft skills e as respectivas frequências com que estes aparecem nas descrições das vagas de gerentes de projetos de TI extraídas do Linkedln, o que levou a inferir sobre padrões de comportamento nos dados.

Além da análise de frequência, realizou-se também a análise de conteúdo a partir das descrições de vagas de gerentes de projetos de TI do Linkedln. Uma das vantagens da análise de conteúdo é que ela é discreta e não reativa (Marshall \& Rossman, 2006) e pode ser conduzida sem interferir no meio estudado. Uma desvantagem seria a inferência que o pesquisador pode fazer com base em sua interpretação do material estudado. A ferramenta usada na análise de dados do Linkedln foi o Excel.

Diante da base de dados com 1.368 descrições de vagas de gerentes de projetos, o passo seguinte foi a eliminação das vagas duplicadas. Para isso, foi usado o recurso do próprio Excel, chamado: Remover Duplicatas. Com esta ação, 231 vagas repetidas foram eliminadas, culminando no novo total de 1.137 vagas. Os pesquisadores procederam, então, à leitura completa das 1.137 descrições de vagas para capturar os soft skills mencionados nelas. Ao longo dessa leitura, verificou-se que havia vagas que não eram do segmento de $\mathrm{TI}$ - alvo desta pesquisa - e, portanto, 345 vagas foram excluídas da amostra. Em paralelo com esta leitura, foram descartadas as vagas cujo idioma fosse diferente do português. Os autores se resguardaram a esse direito de analisar somente vagas em português por se tratar de seu idioma nativo. A avaliação em outros idiomas iria requerer uma tradução que poderia causar prejuízos à interpretação e à análise das vagas. Sendo assim, 117 vagas em inglês e mais 4 vagas em espanhol não fizeram parte da análise dos dados desta pesquisa. 0 novo total geral, portanto, para a base de dados final, ficou em 671 vagas. A Tabela 5 demonstra essa variação na quantidade de vagas ocasionada pela higienização da base de dados. 
Tabela 5.

Detalhamento da quantidade de vagas iniciais até chegar à quantidade final

\begin{tabular}{l|l}
\hline Quantidade de Vagas & Valor \\
\hline Vagas iniciais & 1.368 \\
\hline Vagas duplicadas & 231 \\
\hline Vagas excluídas (por não ser de gerente de projetos de TI) & 345 \\
\hline Vagas em inglês excluídas & 117 \\
\hline Vagas em espanhol excluídas & 4 \\
\hline Vagas finais & $\mathbf{6 7 1}$ \\
\hline
\end{tabular}

Fonte: Elaborada pelos autores.

O processo pautou-se na leitura pelos pesquisadores das 671 descrições de vagas que, ao se deparar com uma citação de soft skill, eram copiadas e coladas em uma nova planilha eletrônica manualmente. Nesta nova planilha, uma lista com 326 ocorrências de soft skills (com repetição) foi construída. Uma segunda lista foi criada após a renomeação de soft skills iguais ou semelhantes com a intenção de realizar um agrupamento. Por exemplo, o soft skill "capacidade de liderança" foi renomeado para "liderança", já o soft skill "comunicativo" mudou para "comunicação" e o soft skill "proativo" foi renomeado para "proatividade". Nota-se que as categorias de soft skills aqui utilizadas partiram de um estudo prévio na literatura, sendo que a classificação seguiu uma orientação da teoria, ou estudos já realizados, para análise da realidade estudada.

\section{RESULTADOS E DISCUSSÃO}

A análise das 671 descrições de vagas de gerentes de projetos de TI publicadas no site Linkedln preconiza alguns achados, no tocante aos soft skills, que são apresentados nesta seção.

\subsection{TÍTULOS DAS VAGAS DE GERENTES DE PROJETOS DE TI}

Em relação ao título das vagas, tem-se que o termo mais utilizado foi "Gerente de Projetos", com 493 aparições, representando $73,47 \%$ do total das 671 vagas. Em segundo lugar, vem o termo "Coordenador de Projetos", com 111 ocorrências e representatividade de 16,54\%. Ocupando a terceira posição, aparece o termo "Líder de Projetos" em 31 descrições de vagas, representando 4,62\% do total. Em quarto lugar, apareceu o termo "Project Manager", com 14 manifestações (após a eliminação das vagas descritas em inglês), representando 2,09\%. Em quinto lugar, vem o termo "Analista de Projetos", que aparece em 5 vagas, representando apenas 0,75\%. Todos os outros demais termos: "analista de sistemas", "coordenador de TI", "analista de negócios", "tech lead", "preposto de leiloeiro", "product manager", "analista de inovação", "coordenador de sistemas", "controller", "analista de suporte", "GMO", "consultor" e "gerente de Tl" somaram 16 aparições, representando 2,53\% do total de vagas.

É importante ressaltar que, ao realizar uma pesquisa por vagas no Linkedln, o termo usado na busca é pesquisado, não só no título da vaga, mas também na sua descrição. Isso ajuda a explicar o porquê de as vagas com títulos singulares aparecerem nesta pesquisa, tais como: "preposto de leiloeiro", "analista de suporte", "GMO", entre outras. Portanto, fica dessa forma caracterizada uma condição inalterável do próprio motor de pesquisa da ferramenta.

A análise dos títulos das vagas leva a crer que o termo "gerente de projetos" seja realmente o mais difundido no site de vagas Linkedln. Entretanto, outras vagas de gerentes de projetos referentes a outros termos podem ser olvidadas dos candidatos. É útil que 0 candidato à vaga de gerente de projetos realize a pesquisa também pelas expressões de busca, como: coordenador de projetos, líder de projetos e project manager. A Tabela 6 mostra o detalhe da frequência dos termos usados nos títulos das vagas de gerentes de projetos de TI. 
Tabela 6.

Frequência dos termos usados nos títulos das vagas de gerentes de projetos de TI

\begin{tabular}{l|l|l}
\hline Termos usados nos títulos das vagas & Quantidade & Porcentagem \\
\hline Gerente de projetos & 493 & $73,47 \%$ \\
\hline Coordenador de Projetos & 111 & $16,54 \%$ \\
\hline Líder de Projetos & 31 & $4,62 \%$ \\
\hline Project Manager & 14 & $2,09 \%$ \\
\hline Analista de Projetos & 5 & $0,75 \%$ \\
\hline Analista de Sistemas & 2 & $0,30 \%$ \\
\hline Coordenador de TI & 2 & $0,30 \%$ \\
\hline Analista de Negócios & 2 & $0,30 \%$ \\
\hline Tech Lead & 1 & $0,30 \%$ \\
\hline Preposto de Leiloeiro & 1 & $0,15 \%$ \\
\hline Product Manager & 1 & $0,15 \%$ \\
\hline Analista de Inovação & 1 & $0,15 \%$ \\
\hline Coordenador de Sistemas & 1 & $0,15 \%$ \\
\hline Controller & 1 & $0,15 \%$ \\
\hline Analista de Suporte & 1 & $0,15 \%$ \\
\hline GMO & 1 & $0,15 \%$ \\
\hline Consultor & 1 & $0,15 \%$ \\
\hline Total Geral & 1 & $0,15 \%$ \\
\hline Fonte El & 1 & $100 \%$ \\
\hline
\end{tabular}

Fonte: Elaborada pelos autores.

\subsection{DESCRIÇÕES DAS VAGAS DE GERENTES DE PROJETOS DE TI}

A leitura das 671 descrições de vagas de gerentes de projetos de TI extraídas do site Linkedln propiciou o levantamento dos soft skills mais requisitados pelas empresas referentes às condições desta pesquisa. Em decorrência desta análise, os resultados mostraram que 44 diferentes soft skills do gerente de projetos de TI foram mencionados ao longo da leitura de 617 vagas. Os dez soft skills mais bem ranqueados representam $63,5 \%$ do total e são eles: $1^{\circ}$ - Comunicação; $2^{\circ}$ - Liderança; $3^{\circ}$ Negociação; $4^{\circ}$ Relacionamento interpessoal; $5^{\circ}$ Gestão de conflitos; $6^{\circ}$ Organização; $7^{\circ}$ - Proatividade; $8^{\circ}$ - Trabalho em equipe; $9^{\circ}$ - Solução de problemas e $10^{\circ}$. Flexibilidade. $O$ ranking foi elaborado com base na frequência da aparição dos soft skills nas descrições das vagas de gerentes de projetos de TI no Linkedln, conforme pode ser visto na Tabela 7.

Tabela 7.

Frequência dos soft skills nas vagas de gerentes de projetos de TI do Linkedln

\begin{tabular}{c|l|l|l}
\hline Ranking & Soft Skills & Frequência dos Soft Skills & Porcentagem \\
\hline $1^{0}$ & Comunicação & 51 & $15,6 \%$ \\
\hline $2^{\circ}$ & Liderança & 35 & $10,7 \%$ \\
\hline $3^{\circ}$ & Negociação & 21 & $6,4 \%$ \\
\hline $4^{\circ}$ & Relacionamento interpessoal & 20 & $6,1 \%$ \\
\hline $5^{\circ}$ & Gestão de conflitos & 15 & $4,6 \%$ \\
\hline $6^{0}$ & Organização & 14 & $4,3 \%$ \\
\hline $7^{0}$ & Proatividade & 14 & $4,3 \%$ \\
\hline $8^{\circ}$ & Trabalho em equipe & 14 & $4,3 \%$ \\
\hline $9^{\circ}$ & Solução de problemas & 13 & $4,0 \%$ \\
\hline $10^{\circ}$ & Flexibilidade & 10 & $3,1 \%$ \\
\hline $11^{0}$ & Habilidade de motivar & 9 & $2,8 \%$ \\
\hline $12^{0}$ & Trabalhar sob pressão & 8 & $2,5 \%$ \\
\hline $13^{\circ}$ & Comprometimento com o projeto & 8 & $2,5 \%$ \\
\hline $14^{\circ}$ & Gestão do cliente & 8 & $2,5 \%$ \\
\hline $15^{\circ}$ & Senso crítico & 7 & $2,1 \%$ \\
\hline $16^{0}$ & Autogestão & 7 & $2,1 \%$ \\
\hline
\end{tabular}




\begin{tabular}{|c|c|c|c|}
\hline Ranking & Soft Skills & Frequência dos Soft Skills & Porcentagem \\
\hline $17^{\circ}$ & Atitude & 5 & $1,5 \%$ \\
\hline $18^{\circ}$ & Dinamismo & 5 & $1,5 \%$ \\
\hline $19^{\circ}$ & Aprendizado & 5 & $1,5 \%$ \\
\hline $20^{\circ}$ & Resiliência & 5 & $1,5 \%$ \\
\hline $21^{\circ}$ & Energia & 4 & $1,2 \%$ \\
\hline $22^{\circ}$ & Comportamento ético & 4 & $1,2 \%$ \\
\hline $23^{\circ}$ & Senso de urgência & 4 & $1,2 \%$ \\
\hline $24^{\circ}$ & Construção de equipe & 4 & $1,2 \%$ \\
\hline $25^{\circ}$ & Disciplina & 4 & $1,2 \%$ \\
\hline $26^{\circ}$ & Tomada de decisão & 3 & $0,9 \%$ \\
\hline $27^{\circ}$ & Adaptabilidade & 3 & $0,9 \%$ \\
\hline $28^{\circ}$ & Habilidade de persistir & 3 & $0,9 \%$ \\
\hline $29^{\circ}$ & Habilidade em ser objetivo & 3 & $0,9 \%$ \\
\hline $30^{\circ}$ & Influência & 2 & $0,6 \%$ \\
\hline $31^{\circ}$ & Disponibilidade em servir & 2 & $0,6 \%$ \\
\hline $32^{\circ}$ & Autoridade & 2 & $0,6 \%$ \\
\hline $33^{\circ}$ & Empatia & 2 & $0,6 \%$ \\
\hline $34^{\circ}$ & Autocontrole emocional & 2 & $0,6 \%$ \\
\hline $35^{\circ}$ & Desenvolvimento de pessoas & 1 & $0,3 \%$ \\
\hline $36^{\circ}$ & Habilidade de ouvir & 1 & $0,3 \%$ \\
\hline $37^{\circ}$ & Construção de engajamento & 1 & $0,3 \%$ \\
\hline $38^{\circ}$ & Articulação & 1 & $0,3 \%$ \\
\hline $39^{\circ}$ & Competitividade & 1 & $0,3 \%$ \\
\hline $40^{\circ}$ & Repasse do conhecimento & 1 & $0,3 \%$ \\
\hline $41^{\circ}$ & Construir confiança & 1 & $0,3 \%$ \\
\hline $42^{\circ}$ & Facilitador & 1 & $0,3 \%$ \\
\hline $43^{\circ}$ & Praticidade & 1 & $0,3 \%$ \\
\hline \multirow[t]{2}{*}{$44^{\circ}$} & Habilidade de empreender & 1 & $0,3 \%$ \\
\hline & Total Geral & 326 & $100,0 \%$ \\
\hline
\end{tabular}

Fonte: Elaborada pelos autores.

Dos 671 anúncios de vagas analisados, somente 92 vagas solicitavam pelo menos um soft skill para a posição de gerente de projetos de TI. Isso é o mesmo que dizer que apenas $13,7 \%$ das vagas declaram os soft skills exigidos pelo recrutador.

\subsection{DISCUSSÃO}

Esta seção tem como objetivo apresentar e discutir a comparação dos soft skills encontrados na literatura com os soft skills das vagas de gerentes de projetos de TI retiradas do Linkedln. $\mathrm{O}$ artigo escolhido para a base desta comparação foi o trabalho de Stevenson e Starkweather (2010), conforme descrito na seção de Referencial Teórico. As vagas de gerentes de projetos de TI, em âmbito nacional, extraídas do Linkedln e apresentadas na subseção anterior são 0 outro fator de comparação.

A pesquisa de Stevenson e Starkweather (2010) elenca as competências mais valorizadas na contratação do gerente de projetos sob o ponto de vista dos executivos de TI. O trabalho destes pesquisadores foi realizado em duas etapas. Na primeira, os recrutadores de TI, por meio de survey on-line, elegeram os 15 critérios mais relevantes nos candidatos em potencial, conforme demonstrado em ordem alfabética na Tabela 8.

Tabela 8.

Competências do gerente de projetos de TI por Stevenson e Starkweather (2010)

\begin{tabular}{c|l}
\hline $\mathbf{N}^{\mathbf{0}}$ & Competências \\
\hline 1 & Ajuste cultural \\
\hline 2 & Atitude \\
\hline
\end{tabular}


(Conclusão)

\begin{tabular}{c|l}
\hline $\mathbf{N}^{\mathbf{0}}$ & Competências \\
\hline 3 & Capacidade em lidar com ambiguidade e mudança \\
\hline 4 & Certificação PMP \\
\hline 5 & Conhecimento técnico \\
\hline 6 & Experiência profissional \\
\hline 7 & Formação acadêmica \\
\hline 8 & Habilidade de comunicação em múltiplos níveis organizacionais \\
\hline 9 & Habilidade de escalar \\
\hline 10 & Habilidade escrita \\
\hline 11 & Habilidade verbal \\
\hline 12 & Histórico de trabalho \\
\hline 13 & Liderança \\
\hline 14 & Tamanho de equipes anteriores \\
\hline 15 & Tamanho de projetos anteriores \\
\hline
\end{tabular}

Fonte: Stevenson e Starkweather (2010).

Na segunda etapa, o público-alvo visado pela entrevista foi de executivos de TI de indústrias norteamericanas de diversos segmentos. Tais executivos receberam um questionário via e-mail e, com base em uma escala Likert de 7 pontos, Ihes foi solicitado que ordenassem as competências resultantes da etapa anterior em ordem de importância. Os resultados concluíram que os executivos de TI preferem os soft skills. Os soft skills do gerente de projetos mais importantes, de acordo com o ranking dos executivos de TI da pesquisa de Stevenson e Starkweather (2010), estão listadas na Tabela 9.

Tabela 9.

Soft skills mais valorizados pelos executivos de TI segundo Stevenson e Starkweather (2010)

\begin{tabular}{c|l}
\hline Ranking & Soft skills \\
\hline $1^{\circ}$ & Liderança \\
\hline $2^{\circ}$ & Habilidade de comunicação em múltiplos níveis organizacionais \\
\hline $3^{0}$ & Habilidade verbal \\
\hline $4^{0}$ & Habilidade escrita \\
\hline $5^{\circ}$ & Atitude \\
\hline $6^{\circ}$ & Capacidade em lidar com ambiguidade e mudança \\
\hline
\end{tabular}

Fonte: Stevenson e Starkweather (2010).

Adotar um único termo para cada soft skill é difícil, pois os estudiosos do tema registram uma vasta nomenclatura utilizada para cada soft skill disponível na literatura. Cabe lembrar que neste trabalho buscou-se convergir para termos amplamente compreendidos na literatura, mas que também são reconhecidos pelos profissionais da área, assim sendo estabelecidos previamente. Neste sentido, vale ressaltar que a categorização dos seguintes soft skills: Habilidade de comunicação em múltiplos níveis organizacionais; Habilidade verbal e Habilidade escrita foram agrupadas sob o único soft skill: Comunicação. Outra associação feita foi a do soft skill Capacidade em lidar com ambiguidade e mudança de, Stevenson e Starkweather (2010), com o soft skill identificado pelos autores: Flexibilidade. Essas associações de termos (categorias de análise) foram feitas com base nas definições dos soft skills contidos na Tabela 3Erro! Fonte de referência não encontrada.

As descobertas de Stevenson e Starkweather (2010) convergiram com a presente pesquisa para os soft skills de Liderança e Comunicação. Neste caso, ocorreu apenas uma inversão de primeiro para segundo lugar no ranking entre as duas pesquisas. As descrições das vagas de gerentes de projetos de TI no Brasil retiradas do Linkedln também fizeram referência aos soft skills de Atitude e Flexibilidade que ocuparam as posições 5 e 6 do ranking de Stevenson e Starkweather (2010), mas numa escala de menor importância, por estarem nas $10^{\circ}$ e $17^{\circ}$ posições, respectivamente. A Figura 2 demonstra os dois rankings e as comparações entre eles. 


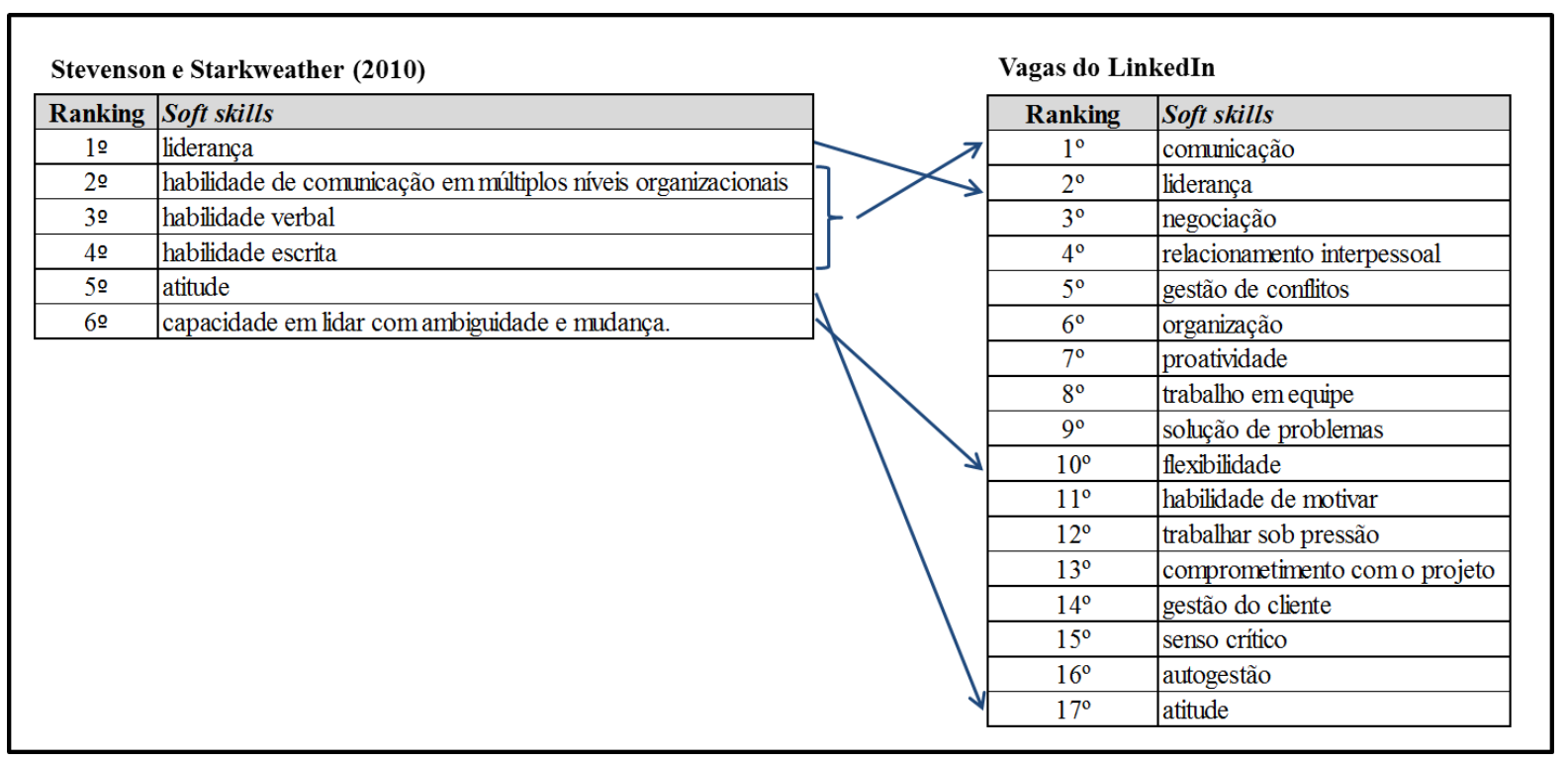

Figura 2. Comparação dos soft skills entre os rankings de Stevenson e Starkweather (2010) e as vagas do Linkedln

Fonte: Elaborada pelos autores com base em Stevenson e Starkweather (2010).

Embora o soft skill Comunicação nesta pesquisa represente o agrupamento de três soft skills da pesquisa de Stevenson e Starkweather (2010), seu posicionamento como número 1 nas descrições de vagas de gerente de projetos de TI do ranking do Linkedln possui congruência com a literatura acadêmica. Berry (1998, p. 1) ressalta que "as habilidades de comunicação são muito mais importantes" para os profissionais do setor de TI. E Sukhoo et al. (2005, p. 694) confirmam dizendo que "Não se pode negar o fato de que o sucesso de qualquer projeto depende do gerenciamento criterioso de todas as informações".

Além disso, Kliem (1991, p. 78) preconiza que os gerentes de projetos devem possuir seis importantes categorias de soft skills e contextualiza a categoria Comunicação conforme a seguir: "Os gerentes de projetos deveriam ter fortes habilidades de comunicação escrita e oral. Eles devem ser capazes de escrever um documento simples, claro, como um memorando ou uma política para clarificar ou resolver um problema complexo". Neste trecho, é possível depreender que Kliem (1991) entende que as habilidades verbal e escrita compõem o conceito da categoria de soft skill denominado Comunicação. Kliem (1991, p. 78) segue afirmando que: "Sem as habilidades-chave de comunicação, o gerente de projetos pode estar em desvantagem. [...] Isso leva a uma comunicação pobre entre os empregados acima e abaixo da cadeia de comando". Este último trecho fecha o argumento que justifica a associação do soft skill Comunicação desta pesquisa em referência ao soft skill Habilidade de Comunicação em múltiplos níveis organizacionais da pesquisa de Stevenson e Starkweather (2010).

\section{CONCLUSÃO}

A análise de 671 descrições de vagas de gerente de projetos de TI extraídas do Linkedln demonstrou que somente $13,7 \%$ destas vagas citam algum tipo de soft skill em sua descrição. Essa estatística pode ser alvo de estudos futuros por meio de pesquisa investigativa com os recrutadores para identificar os motivos que os levam a não citar os soft skills requeridos nas descrições das vagas de gerentes de projetos de TI.

Como contribuição prática para os gerentes de projetos de $\mathrm{Tl}$, é recomendável que se faça uma autoanálise ou uma consulta com colegas com quem já tenha trabalhado em projetos para identificar seus soft skills faltantes. A sugestão dos pesquisadores é compará-los com os resultados desta pesquisa. Em caso de haver uma lacuna, sugere-se o desenvolvimento de tais habilidades.

A comparação entre as descobertas empíricas desta pesquisa com a literatura usada promoveu 0 desfecho deste artigo. Houve uma harmonização entre a literatura e os resultados deste trabalho. Em tempos de avanços tecnológicos vertiginosos e mudanças frequentes, a manutenção dos hard skills será crucial para os gerentes de projetos, entretanto a demanda pelos soft skills é crescente, porque estimula o crescimento pessoal 
e, consequentemente, 0 aumento de lucros e receitas nas empresas, conforme menciona o relatório do Linkedln (2018).

Este artigo, como ocorre em qualquer pesquisa acadêmica, possui limitações que poderão ser exploradas em pesquisas futuras. $O$ site de vagas usado para a extração das descrições de vagas de gerente de projetos e consequente análise foi o Linkedln. Muito embora esse seja uma das redes sociais de emprego mais consagradas no mundo corporativo, os autores poderiam ter ampliado a coleta de dados para outras plataformas de emprego on-line, visando a uma diversidade na pesquisa.

Este trabalho poderá ser replicado em estudos sobre gerentes de projetos de outros tipos de indústrias, como: construção civil, engenharia, automotiva, além do segmento de TI adotado. Além disso, é possível realizar a mesma pesquisa fora do Brasil, visando comparar os resultados deste país com outros.

\section{REFERÊNCIAS}

Ahmed, F., Capretz, L. F., \& Campbell, P. (2012). Evaluating the demand for soft skills in software development. IT Professional, 14(1), 44-49. https://doi.org/10.1109/MITP.2012.7

Ahsan, K., Ho, M., \& Khan, S. (2013). Recruiting project managers: A comparative analysis of competencies and recruitment signals from job advertisements. Project Management Journal, 44(5), 36-54. https://doi.org/10.1002/pmj.21366

Andoh-Baidoo, F. K., Villarreal, M. A., Koong, K. S., Cornejo, H., Schmidt, N., Colunga, H., \& Mesa, R. (2011). Key competencies for global project managers: A cross cultural study of the UK and India. International Journal of Business and Systems Research, 5(3), 223-243. https://doi.org/10.1504/JJBSR.2011.039294

Balcar, J., Šimek, M., \& Filipová, L. (2018). Soft Skills of Czech Graduates, 18(1), 45-60. https://doi.org/10.2478/revecp-2018-0003

Belzer, K. (2001). Project management: still more art than science. PM Forum Featured Papers, 1-6. Retrieved from http://www.egrupos.net/cgibin/eGruposDMime.cgi?K9U7J9W7U7xumopxCnhuMyqjdkuymCYTUVCvthCnoqdy-qlhhyCXUQkfb7

Berbegal-Mirabent, J., Gil-Doménech, D., \& Alegre, I. (2017). Where to locate? A project-based learning activity for a graduate-level course on operations management. International Journal of Engineering Education, 33(5), 1586-1597.

Bolli, T., \& Renold, U. (2015). Comparative advantages of school and workplace environment in competence acquisition: Empirical evidence from a survey among professional tertiary education, (389).

https://doi.org/10.1108/EBHRM-05-2015-0020

Bullhornreach. (2014). 2014 Global Social Recruiting Activity Report. Www.Bullhornreach.Com, (August).

Retrieved from https://www.bullhorn.com/uk/resources/2014-social-recruiting-activity-report/

Collins, D. (1992). Collins Cobuild Essential English Dictionary.

Creasy, T., \& Anantatmula, V. S. (2013, December). From every direction - How personality traits and dimensions of project managers can conceptually affect project success. Project Management Journal.

https://doi.org/10.1002/pmj.21372

Creswell, J. W. (2010). Projeto de pesquisa: métodos qualitativo, quantitativo e misto (3a. ed.). Artmed.

Danneels, E. (2000). The dynamics of product innovation and firm competencies. In Academy of Management Proceedings 2000 BPS (pp. 1-7).

Dench, S. (1997). Changing skill needs: what makes people employable ? Industrial and Commercial Training, 29(6), 190-193.

El-Sabaa, S. (2001). The skills and career path of an effective project manager. International Journal of Project Management, 19(1), 1-7. https://doi.org/10.1016/S0263-7863(99)00034-4

Elliott, M., \& Dawson, R. (2015). Excellence in IT Project Management - Firing Agile Silver Bullets. International Journal of Human Capital and Information Technology Professionals, 14.

https://doi.org/10.4018/IJHCITP.2015070105 
FEBRABAN. (2017). Pesquisa FEBRABAN de Tecnologia Bancária 2017. Deloitte.

Geithner, S., \& Menzel, D. (2016). Effectiveness of Learning Through Experience and Reflection in a Project Management Simulation. Simulation \& Gaming, 47(2), 228-256. https://doi.org/10.1177/1046878115624312

Girard, A., \& Fallery, B. (2010). Human resource management on Internet: New perspectives. Contemporary Management Research, 4(2), 15.

Gray, K., \& Ulbrich, F. (2017). Ambiguity acceptance and translation skills in the project management literature. International Journal of Managing Projects in Business, 10(2), 423-450. https://doi.org/10.1108/IJMPB-05-20160044

Joseph, D., Ang, S., Chang, R. H. L., \& Slaughter, S. a. (2010). Practical intelligence in IT: Assessing soft skills of IT professionals. Communications of the ACM, 53(2), 149-154. https://doi.org/10.1145/1646353.1646391

Keil, M., Lee, H. K., \& Deng, T. (2013). Understanding the most critical skills for managing IT projects: A Delphi study of IT project managers. Information and Management, 50(7), 398-414.

https://doi.org/10.1016/j.im.2013.05.005

Kliem, R. L. (1991). Choosing the Right Manager for Your Special Project. Computerworld, 25(29), 1991.

Lent, B., \& Pinkowska, M. (2012). Soft skills needed in the ICT project management - classification and maturity level assessment. International Journal of Applied Systemic Studies, 4(3), 168-185.

https://doi.org/10.1504//JASS.2012.051133

Linkedin. (2018). 2018 Workplace Learning Report. Linkedln Learning, 1-51.

Livesey, P. V. (2016). Insights of project managers into the problems in project management. Construction Economics and Building, 16(1), 90-103.

Livesey, P. V. (2017). Goleman-Boyatzis Model of Emotional Intelligence for Dealing with Problems in Project Management. Construction Economics and Building, 17(1), 20. https://doi.org/10.5130/AJCEB.v17i1.5101

Marshall, C., \& Rossman, G. (2006). Data Collection Methods. In Designing qualitative research (pp. 97-141). Sage publications.

Mateo, J. R. S. C., De Navamuel, E. D. R., \& Villa, M. A. G. (2017). Are project managers ready for the 21 th challenges? A review of problem structuring methods for decision support. International Journal of Information Systems and Project Management, 5(2), 43-56. https://doi.org/10.12821/ijispm050203

Miles, M. B., Huberman, A. M., \& Saldaña, J. (1994). Qualitative Data Analysis: An Expanded Sourcebook. In Thousand Oaks, CA: Sage Publications.

Pant, I., \& Baroudi, B. (2008). Project management education: The human skills imperative. International Journal of Project Management, 26(2), 124-128. https://doi.org/10.1016/j.jproman.2007.05.010

Pilati, R., \& Araújo, M. E. B. de O. (2008). Gerenciamento de Impressão nas entrevistas de seleção : proposição de uma agenda de pesquisa. Revista Psicologia Organizações e Trabalho, 8(2), 121-138.

Pinto, J. K., Patanakul, P., \& Pinto, M. B. (2017). "The aura of capability": Gender bias in selection for a project manager job. International Journal of Project Management, 35(3), 420-431.

https://doi.org/10.1016/j.jproman.2017.01.004

Pozzebon, M., \& Petrini, M. (2013). Critérios para Condução e Avaliação de Pesquisas Qualitativas de Natureza Crítico-Interpretativa. Pesquisa Qualitativa Em Administração: Fundamnetos, Métodos e Usos No Brasil., 51-72.

Walter Saunders, J., Nunes, B., \& de Carvalho, M. M. (2018). Project Managers' Competences: What Do Job Advertisements and the Academic Literature Say? Project Management Journal, 49(3), 82-97. https://doi.org/10.1177/8756972818770884

Schoper, Y. G., Wald, A., Ingason, H. T., \& Fridgeirsson, T. V. (2018). Projectification in Western economies: A comparative study of Germany, Norway and Iceland. International Journal of Project Management, 36(1), 71-82. https://doi.org/10.1016/j.jproman.2017.07.008

Skulmoski, G. J., \& Hartman, F. T. (2009). Information systems project manager soft competencies: a projectphase investigation. Project Management Journal, 41(1), 61-80. https://doi.org/10.1002/pmj 
Soares, F. O., Sepúlveda, M. J., Monteiro, S., Lima, R. M., \& Dinis-Carvalho, J. (2013). An integrated project of entrepreneurship and innovation in engineering education. Mechatronics, 23(8), 987-996.

https://doi.org/10.1016/j.mechatronics.2012.08.005

Stevenson, D. H., \& Starkweather, J. A. (2010). PM critical competency index: IT execs prefer soft skills. International Journal of Project Management, 28(7), 663-671. https://doi.org/10.1016/j.jproman.2009.11.008

Sukhoo, A., Barnard, A., Eloff, M., \& Van der Poll, J. A. (2005). Accommodating soft skills in software project management. Issues in Informing Science and Information Technology, 2, 691-703. Retrieved from http://umkndsp01.unisa.ac.za/handle/10500/4919\%5Cnhttp://www.markmcphee.talktalk.net/production_process/papers/soft_ skills_in_project_management.pdf

The Standish Group. (2014). The Standish group: the chaos report. Project Smart, 16. https://doi.org/10.1016/S0895-7061(01)01532-1

Tifferet, S., \& Vilnai-Yavetz, I. (2018). Self-presentation in Linkedln portraits: Common features, gender, and occupational differences. Computers in Human Behavior, 80, 33-48. https://doi.org/10.1016/j.chb.2017.10.013

Turner, M. (2016). Beyond the iron triangle: reflections of an early career academic. International Journal of Managing Projects in Business, 9(4), 892-902. https://doi.org/10.1108/IJMPB-01-2016-0005

Wikle, T. A., \& Fagin, T. D. (2015). Hard and Soft Skills in Preparing GIS Professionals: Comparing Perceptions of Employers and Educators. Transactions in GIS, 19(5), 641-652. https://doi.org/10.1111/tgis.12126

Zahra, S., Nazir, A., \& Khalid, A. (2014). Performing Inquisitive Study of PM Traits Desirable for Project Progress. International Journal of ..., 6(2), 41-47. https://doi.org/10.5815/ijmecs.2014.02. 06 